\title{
Doppler tomography of transiting exoplanets: a prograde, low-inclined orbit for the hot Jupiter CoRoT-11b
}

\author{
D. Gandolfi ${ }^{1}$, A. Collier Cameron ${ }^{2}$, M. Endl ${ }^{3}$, A. F. Lanza ${ }^{4}$, C. Damiani ${ }^{4,5}$, R. Alonso ${ }^{6,7}$, W. D. Cochran ${ }^{3}$, M. Deleuil ${ }^{5}$, \\ M. Fridlund ${ }^{1}$, A. P. Hatzes ${ }^{8}$, and E. W. Guenther ${ }^{8}$
}

\author{
${ }^{1}$ Research and Scientific Support Department, ESTEC/ESA, PO Box 299, 2200 AG Noordwijk, The Netherlands \\ e-mail: davide.gandolfi@esa.int \\ 2 School of Physics \& Astronomy, University of St. Andrews, North Haugh, St. Andrews, Fife KY16 9SS, UK \\ 3 McDonald Observatory, University of Texas at Austin, Austin, TX 78712, USA \\ ${ }^{4}$ INAF - Osservatorio Astrofisico di Catania, via S. Sofia 78, 95123 Catania, Italy \\ 5 Laboratoire d'Astrophysique de Marseille, CNRS \& University of Provence, 38 rue Frédéric Joliot-Curie, \\ 13388 Marseille Cedex 13, France \\ 6 Instituto de Astrofísica de Canarias, C/ Vía Láctea s/n, 38205 La Laguna, Spain \\ 7 Departamento de Astrofísica, Universidad de La Laguna, 38206 La Laguna, Spain \\ 8 Thüringer Landessternwarte, Sternwarte 5, Tautenburg, 07778 Tautenburg, Germany
}

Received 3 May 2012 / Accepted 12 June 2012

\section{ABSTRACT}

\begin{abstract}
We report the detection of the Doppler shadow of the transiting hot Jupiter CoRoT-11b. Our analysis is based on line-profile tomography of time-series, Keck/HIRES high-resolution spectra acquired during the transit of the planet. We measured a sky-projected, spin-orbit angle $\lambda=0.1^{\circ} \pm 2.6^{\circ}$, which is consistent with a very low-inclined orbit with respect to the stellar rotation axis. We refined the physical parameters of the system using a Markov chain Monte Carlo simultaneous fitting of the available photometric and spectroscopic data. An analysis of the tidal evolution of the system shows how the currently measured obliquity and its uncertainty translate into an initial absolute value of less than about $10^{\circ}$ on the zero-age main sequence, for an expected average modified tidal quality factor of the $\operatorname{star}\left\langle Q_{\star}^{\prime}\right\rangle \gtrsim 4 \times 10^{6}$. This is indicative of an inward migration scenario that would not have perturbed the primordial low obliquity of CoRoT-11b. Taking into account the effective temperature and mass of the planet host $\operatorname{star}\left(T_{\text {eff }}=6440 \mathrm{~K}\right.$, $M_{\star}=1.23 M_{\odot}$ ), the system can be considered a new telling exception to the recently proposed trend, according to which relatively hot and massive stars $\left(T_{\text {eff }}>6250 \mathrm{~K}, M_{\star}>1.2 M_{\odot}\right)$ seem to be preferentially orbited by hot Jupiters with high obliquity.
\end{abstract}

Key words. planet-star interactions - planet-disk interactions - planetary systems - binaries: eclipsing - stars: individual: CoRoT-11

\section{Introduction}

Time-series, high-resolution spectroscopic observations of planetary transits allow us to detect the Rossiter-McLaughlin (RM) effect (Rossiter 1924; McLaughlin 1924) and to measure the skyprojected system obliquity $\lambda$, i.e., the angle in the plane of the sky between the projections of the planet's orbital angular momentum and the star's rotation spin. When a planet transits in front of its host star, it subsequently occults different parts of the stellar disc. For a rotating star, this results in a distortion of the stellar line profiles that change during the transit. If stellar rotation is the dominant source of line-broadening, high-resolution spectroscopy can resolve the stellar line profiles and shows the distortion (bump) moving across the line profiles, which is the result of the missing starlight blocked by the transiting planet (e.g., Collier Cameron et al. 2010a,b; Miller et al. 2010). On the other hand, if stellar rotation is not the dominant broadening mechanism, the RM effect is detected as an anomalous Doppler shift of the stellar lines (e.g., Queloz et al. 2000; Ohta et al. 2005; Giménez 2006; Gaudi \& Winn 2007; Winn 2010).

Giant planets with a semi-major axis $a \lesssim 0.1$ AU (hot Jupiters) are thought to have formed at large orbital distances from their parent star and then migrated inwards to their current position through a) tidal interaction with the proto-planetary disc in the pre-main sequence phase of the star/planet evolution (e.g., Lin et al. 1996); b) planet-planet scattering (e.g., Rasio \& Ford 1996); c) gravitational interaction with a third outer planet or stellar companion (Kozai mechanism; e.g. Kozai 1962; Fabrycky \& Tremaine 2007; Wu et al. 2007; Naoz et al. 2011). The migration mechanism is not completely understood yet and the proposed theories predict different final obliquities with low values in the case of a migration governed by planet-disc interaction and significant misalignment in the case of the other mechanisms (Triaud et al. 2010; Morton \& Johnson 2011). Measuring the spin-orbit obliquity of hot Jupiters can thus provide insights into their migration mechanism and help to discern between the rivaling theories.

Winn et al. (2010) empirically found that hot Jupiters orbiting stars with an effective temperature $T_{\text {eff }} \geq 6250 \mathrm{~K}$ and a mass $M_{\star} \geq 1.2 M_{\odot}$ tend to be misaligned. A similar result was obtained by Schlaufman (2010), who found evidence of spinorbit misalignment along the line of sight in systems with massive stars and planets. According to Winn et al. (2010), either a planet formation scenario dependent on the stellar mass, or an efficient tidal realignment of hot Jupiters around cool stars, might account for the suspected trend. More recently, Triaud (2011) suggested that the relatively rapid evolution of stars with $M_{\star} \geq 1.2 M_{\odot}$ combined with an observational bias, might 
explain the lack of aligned systems for stars with $T_{\text {eff }} \geq 6250 \mathrm{~K}$. However, the number of exoplanets with secure measurements of the $\lambda$ angle is not yet statistically significant enough to confirm this trend, as pointed out by Moutou et al. (2011).

Gandolfi et al. (2010) announced the discovery of the transiting hot Jupiter CoRoT-11b, a 2.33 $M_{\text {Jup }}$ planet in a 2.99 days orbit around a $T_{\text {eff }}=6440 \mathrm{~K}$ dwarf $\operatorname{star}(\mathrm{SpT}=\mathrm{F} 6 \mathrm{~V})$ with a relatively high projected rotational velocity $\left(v \sin i_{\star}=40 \mathrm{~km} \mathrm{~s}^{-1}\right)$. With the aim of detecting the RM effect of the planet, the authors acquired radial velocity (RV) measurements during the transit, using the High Resolution Echelle Spectrometer (HIRES) mounted on the Keck I $10 \mathrm{~m}$ telescope, at the Keck Observatory (Mauna Kea, Hawai'i). Unfortunately, the observations were scheduled according to an old, slightly incorrect transit ephemeris, resulting in a partial coverage of the event. Although the HIRES RV data clearly show the RM anomaly of CoRoT-11, Gandolfi et al. (2010) were unable to place strong constraints on the orbit obliquity, owing to the partial coverage of the transit event and the relatively high $v \sin i_{\star}$ of the star, which strongly affected the precision of their RV measurements.

In this Letter, we show how the problems encountered in deriving accurate RV measurements of rapidly rotating stars to assess the shape of their RM anomaly can be overcome with Doppler imaging techniques, even in the occurrence of a partial spectroscopic coverage of the transit event. On the basis of line-profile tomography applied to the above-mentioned HIRES spectra, we succeed in detecting the Doppler shadow of CoRoT-11b and measuring the sky-projected obliquity of the system. A simultaneous reanalysis of the available photometric and spectroscopic data using a Markov chain Monte Carlo (MCMC) approach, enabled us to refine the system's parameters.

\section{Observations and data analysis}

As part of NASA's key science project to support the CoRoT mission, high-resolution $(R \approx 50000)$ transit spectroscopy was carried out using the HIRES spectrograph (Vogt et al. 1994). A detailed description of the adopted instrument set-up and data reduction was reported in Gandolfi et al. (2010). Twelve consecutive spectra of $900 \mathrm{~s}$ each were gathered during the transit that occurred on the night of 1 July 2009. The observations started about $1.2 \mathrm{~h}$ before the beginning of the transit and erroneously stopped $\sim 45 \mathrm{~min}$ before the end of the event, owing to an incorrect transit ephemeris at the time of the observations. The altitude of CoRoT-11 increased during the time-series observations, with an airmass varying from 1.80 to 1.05 . Two more out-oftransit spectra were secured on the night before and one on the same night, to properly combine the HIRES RV measurements with data previously taken with other instruments.

The HIRES spectra were acquired using an $I_{2}$ absorption cell to track any instrumental drifts and improve the RV precision. Since our analysis is based on Doppler imaging of the stellar line profile, we used only the echelle orders that were not contaminated by the $I_{2}$ absorption lines. The first spectrum was discarded as affected by the high airmass of the exposure. This resulted in a set of eleven consecutive HIRES spectra covering the wavelength range $3800-4860 \AA$, with a signal-to-noise ratio ranging from 40 to 70 per pixel at $4300 \AA$.

For each exposure, a composite stellar line profile was computed using the least squares deconvolution (LSD) algorithm of Donati et al. (1997). This is the line profile that, when convolved with a mask of delta functions at the wavelengths of known lines, with relative depths computed for an F6 V spectrum
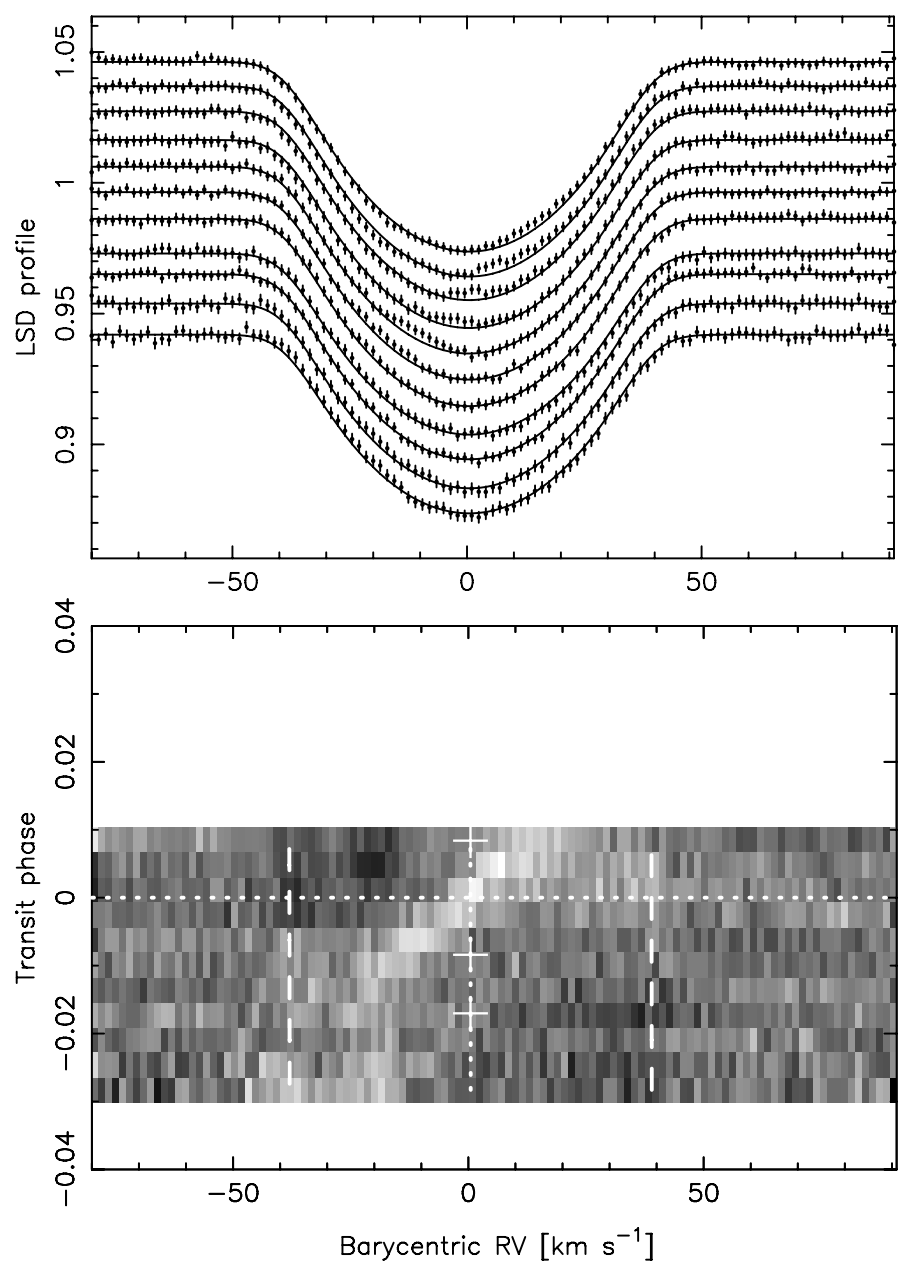

Fig. 1. Upper panel: least squares, deconvolved line profiles of CoRoT-11 (points with error bars), as obtained from the time-series HIRES spectra acquired during the transit of the planet. The limbdarkened, rotationally-broadened line profile of the best fitting model is shown with solid lines. Radial velocity (wavelength) increases from left to right, time from bottom to top. The planet signature is the bump that migrates from about $-20 \mathrm{~km} \mathrm{~s}^{-1}$ near the middle of the time series, to about $+15 \mathrm{~km} \mathrm{~s}^{-1}$ in the upper line profile. Lower panel: residuals of the composite spectral line profiles of CoRoT-11 following the subtraction of the above-mentioned synthetic profile. Orbital phase increases vertically. The two vertical dashed lines mark the $\pm v \sin i_{\star}$ of the star. The three crosses denotes the first, second, and third planet's contacts. A moderately bright feature, "travelling" from the bottom left to the top right corner, appears in the residual map as the result of the transit of the planet across the stellar disc.

using a Kurucz atmosphere model (Kurucz 1979), yields an optimal inverse-variance-weighted fit to the observed spectral orders. The stellar line mask included 1140 lines in the 15 HIRES echelle orders spanning the wavelength range from $4016 \AA$ to $4856 \AA$. The deconvolved profiles were computed on a linear velocity scale in the barycentric frame, with a velocity increment of $1.5 \mathrm{~km} \mathrm{~s}^{-1}$ pixel $^{-1}$ (Fig. 1, upper panel).

The lower panel of Fig. 1 shows the time-series residuals of the composite spectral line profile in grey-scale form, following subtraction of the the limb-darkened, rotationally-broadened line profile of the best-fitting stellar model. The trailed spectrum reveals a narrow, moderately bright feature moving with a constant radial acceleration through the composite stellar line profile from the bottom left to the top right corner. This feature is identifiable as the spectral signature of the photospheric light 
D. Gandolfi et al.: A prograde, low-inclined orbit for the hot Jupiter CoRoT-11b

Table 1. CoRoT-11 system parameters as derived from our MCMC analysis and comparison with the values reported in the literature.

\begin{tabular}{|c|c|c|c|c|c|}
\hline Parameter & Symbol & Gandolfi et al. (2010) & Southworth (2011) & This work & Unit \\
\hline Orbital period & $P$ & $2.994330 \pm 0.000011$ & - & $2.994325 \pm 0.000021$ & days \\
\hline Transit epoch & $T_{0}$ & $2454597.6790 \pm 0.0003$ & - & $2454690.49838 \pm 0.00016$ & days \\
\hline Transit duration & $d_{\mathrm{tr}}$ & $2.5009 \pm 0.0144$ & - & $2.44488 \pm 0.01800$ & hours \\
\hline Impact parameter & $b$ & $0.8180 \pm 0.0080$ & - & $0.8108 \pm 0.0077$ & $R_{\star}$ \\
\hline Planet-to-star area ratio & $\left(R_{\mathrm{p}} / R_{\star}\right)^{2}$ & $0.011449 \pm 0.000107$ & $0.011381 \pm 0.000483$ & $0.011600 \pm 0.000230$ & \\
\hline Scaled star radius & $R_{\star} / a$ & $0.1451 \pm 0.0017$ & $0.1452 \pm 0.0022$ & $0.1416 \pm 0.0024$ & \\
\hline Orbit inclination & $i_{\mathrm{p}}$ & $83.17 \pm 0.15$ & $83.13 \pm 0.19$ & $83.41 \pm 0.17$ & deg \\
\hline Orbit semi-major axis & $a$ & $0.0436 \pm 0.005$ & $0.0440 \pm 0.0016$ & $0.04351 \pm 0.00036$ & $\mathrm{AU}$ \\
\hline Orbit eccentricity & $e$ & 0 (fixed) & 0 (fixed) & 0 (fixed) & \\
\hline RV semi-amplitude & $K$ & $280.0 \pm 40.0$ & - & $304.0 \pm 32.0$ & $\mathrm{~m} \mathrm{~s}^{-1}$ \\
\hline Systemic radial velocity ${ }^{a}$ & $\gamma$ & $-1.336 \pm 0.044$ & - & $-1.342 \pm 0.041$ & $\mathrm{~km} \mathrm{~s}^{-1}$ \\
\hline Star mass & $M_{\star}$ & $1.27 \pm 0.05$ & $1.26 \pm 0.14$ & $1.23 \pm 0.03$ & $M_{\odot}$ \\
\hline Star radius & $R_{\star}$ & $1.37 \pm 0.03$ & $1.374 \pm 0.061$ & $1.33 \pm 0.04$ & $R_{\odot}$ \\
\hline Star mean density & $\rho_{\star}$ & $0.69 \pm 0.02$ & $0.69 \pm 0.03$ & $0.74 \pm 0.04$ & $\mathrm{~g} \mathrm{~cm}^{-3}$ \\
\hline Star projected rotational velocity & $v \sin i_{\star}$ & $40 \pm 5$ & - & $38.47 \pm 0.07$ & $\mathrm{~km} \mathrm{~s}^{-1}$ \\
\hline Sky-projected spin-orbit angle & $\lambda$ & - & - & $0.1 \pm 2.6$ & $\operatorname{deg}$ \\
\hline Planet mass & $M_{\mathrm{p}}$ & $2.33 \pm 0.34$ & $2.34 \pm 0.39$ & $2.49 \pm 0.27$ & $M_{\text {Jup }}$ \\
\hline Planet radius & $R_{\mathrm{p}}$ & $1.43 \pm 0.03$ & $1.426 \pm 0.057$ & $1.390 \pm 0.033$ & $R_{\text {Jup }}$ \\
\hline Planet density & $\rho_{\mathrm{p}}$ & $0.99 \pm 0.15$ & $1.01 \pm 0.16$ & $0.93 \pm 0.12$ & $\mathrm{~g} \mathrm{~cm}^{-3}$ \\
\hline Equilibrium temperature & $T_{\mathrm{eq}}$ & $1657 \pm 55$ & $1735 \pm 34$ & $1715 \pm 36$ & $\mathrm{~K}$ \\
\hline
\end{tabular}

Notes. ${ }^{(a)}$ As derived from the HARPS/Standard RV measurements.

blocked by CoRoT-11b as it transits in front of the stellar disc. Important results can be drawn from a qualitative inspection of the plot in Fig. 1. First, the planet's Doppler shadow appears over the approaching limb of the star and moves towards the receding half, confirming the prograde orbit scenario suggested by the shape of the observed RV anomaly (Gandolfi et al. 2010). The absolute value of the radial velocity of the light blocked by the planet at the beginning of the transit is $\sim 20 \mathrm{~km} \mathrm{~s}^{-1}$, which agrees with the expected value for a planetary transit with an impact parameter $b=0.81$ and for a stellar projected rotational velocity $v \sin i_{\star}=40 \mathrm{~km} \mathrm{~s}^{-1}$ (see Table 1). At the phase of mid-transit, the trail has a zero barycentric velocity relative to the star, i.e., the planet crosses the sky-projected stellar spin axis at the midpoint of the transit. Taking into account the non-central planetary transit $(b \neq 0)$, this indicates that the CoRoT-11 system is closely aligned in the plane of the sky $\left(\lambda \simeq 0^{\circ}\right)$.

The time-series spectra were modelled together with the CoRoT photometry and the out-of-transit RV data from HIRES, HARPS, SOPHIE, and TLS/Coudé, as listed in Gandolfi et al. (2010). The system parameters were computed using the MCMC approach described by Collier Cameron et al. (2010a), in which the photometry, the out-of-transit RV data, and the least squares deconvolved rotation profiles are fitted simultaneously.

The photometric transit model employs the algorithm of Mandel \& Agol (2002) in the small-planet approximation, using a four-coefficient non-linear limb-darkening model with coefficients interpolated from the tabulation of Claret (2004) to match the CoRoT bandpass for a star with the same effective temperature, metallicity, and surface gravity as CoRoT-11. The light contamination fraction of $13.0 \pm 1.5 \%$ (Gandolfi et al. 2010) was taken into account in modelling the CoRoT photometry. The modelling procedure is described by Collier Cameron et al. (2007) and Pollacco et al. (2008), and yields five parameters: the orbital period $P$, the epoch $T_{0}$ of mid-transit, the planet-to-star area ratio $\left(R_{\mathrm{p}} / R_{\star}\right)^{2}$, the scaled stellar radius $R_{\star} / a$ as a fraction of the orbit's semi-major axis $a$, and the dimensionless impact parameter $b=a \cos i_{\mathrm{p}} / R_{\star}$, where $i_{\mathrm{p}}$ is the inclination of the planet's orbital axis to the line of sight. The stellar mass is estimated at each time step using the empirical calibration of Enoch et al. (2010), based on the eclipsing-binary data compilation of Torres et al. (2010). The out-of-transit RV curve was fitted using a circular orbit model of velocity amplitude $K$. A separate zero-point velocity was fitted for each of the different RV data sets.

For the line-profile analysis during the transit, we used the line-profile decomposition model of Collier Cameron et al. (2010a), in which the stellar profile is modelled as the convolution of a narrow Gaussian of width $v_{\mathrm{FWHM}}$ (representing the combined instrumental and intrinsic stellar photospheric line profile) and a limb-darkened rotation profile of width $v \sin i_{\star}$. The light blocked by the planet was treated as a Gaussian of the same width as the intrinsic profile, whose strength was modulated using the model of Mandel \& Agol (2002). The trajectory of the planet's signature in velocity space is governed by the projected spin-orbit misalignment angle $\lambda$, the stellar $v \sin i_{\star}$, the stellar radial acceleration, the transit impact parameter $b$, and the zero-point of the velocity scale on the LSD profile $\left(v_{0}\right)$. The latter parameter may differ significantly from the systemic velocity $\gamma$ established by the out-of-transit RV measurements, as it depends on the form of the stellar spectrum and the line weights and wavelengths used in the LSD procedure. In all, the trailed spectrogram was modelled as a function of four new MCMC parameters $\left(\lambda, v \sin i_{\star}, v_{\mathrm{FWHM}}\right.$, and $\left.v_{0}\right)$, in addition to the RV curve semi-amplitude $K$ (which is also constrained by the RV solution) and the impact parameter $b$ (which is also determined by the photometry). Figure 2 shows the correlation diagrams for the joint posterior probability distributions of the four tomography parameters, as derived from our MCMC analysis. The derived system parameters and their uncertainties are listed in Table 1.

\section{Discussion}

After HD189733b (Collier Cameron et al. 2010a), WASP-33b (Collier Cameron et al. 2010b), and WASP-3b (Miller et al. 2010), CoRoT-11b is the fourth transiting exoplanet for which the $\lambda$ angle has been derived using a Doppler imaging technique. This Letter has demonstrated once more the advantage of this 


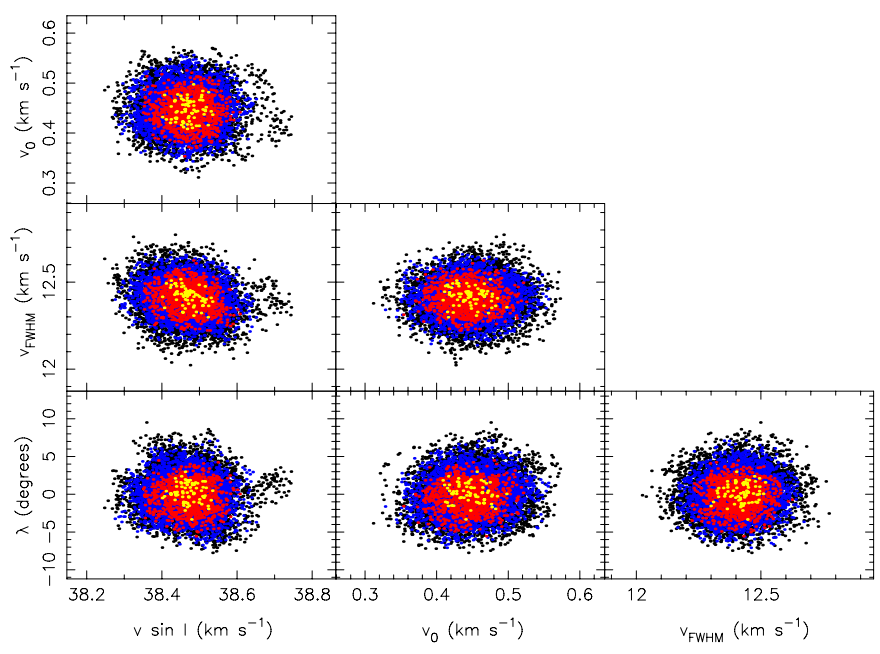

Fig. 2. Correlation diagrams for the joint posterior probability distributions of the four tomography parameters, namely $\lambda, v \sin i_{\star}, v_{\mathrm{FWHM}}$, and $v_{0}$. The colours of the dots reflect the $\Delta \chi^{2}$ of each point, with thresholds at $2.30,6.17$, and 11.8 , to show the two-parameter one, two, and threesigma confidence regions in yellow, red, and blue, respectively (see the online edition of the journal for a color version of this figure).

method relative to standard RV approaches for the analysis of the RM anomaly, especially in cases where accurate RV measurements are rendered unfeasible by the rapid stellar rotation rate. The RM effect of CoRoT-11b was first observed by Gandolfi et al. (2010), who detected the RV anomaly during the transit of the planet. Although the shape of the RV anomaly was clearly indicative of a prograde orbit, the sky-projected obliquity of the system could not be constrained from the RV data. The measurements presented in Gandolfi et al. (2010) did not cover the entire transit and were strongly affected by the high $v \sin i_{\star}$ of the star, preventing any accurate modelling of the RV anomaly. We reanalysed the existing on-transit spectra with the line-profile tomography technique described in Collier Cameron et al. (2010b) and confirmed a prograde orbit for CoRoT-11b. By tracking the trajectory of the missing starlight across the line profile, we measured the sky-projected system's obliquity $\lambda=0.1^{\circ} \pm 2.6^{\circ}$. The advantage of tracking the Doppler shadow of a transiting planet compared to modelling the RV anomaly occurring during the $\mathrm{RM}$ effect, is that the planet's trail is a straight line and its position can be extrapolated even with a partial spectroscopic coverage of the transit. Modelling the RV anomaly can lead to a degeneracy of the solution, especially if the RV data are noisy and do not cover the entire event.

A combined MCMC analysis of the available CoRoT photometry and spectroscopic data, yields the system parameters listed in Table 1. A comparison with the previous values reported in the discovery paper and those published recently by Southworth (2011), illustrates the very good agreement (within $1 \sigma$ ) of the three sets of results (Table 1).

The RM effect allows us to measure only the sky-projected angle $\lambda$. The true obliquity $\psi$ between the stellar spin axis and the orbital angular momentum can be derived only if one also knows the inclinations of the planetary orbit $i_{\mathrm{p}}$ and stellar spin axis $i_{\star}$ with respect to the line of sight. While the transit lightcurve modelling provides the former angle, the inclination $i_{\star}$ can be derived from the projected rotational velocity $v \sin i_{\star}$ and stellar radius $R_{\star}$, once the rotation period of the star $P_{\text {rot }}$ is known. Unfortunately, no evidence of spot-induced rotational modulation has been found in the CoRoT light curve, which is

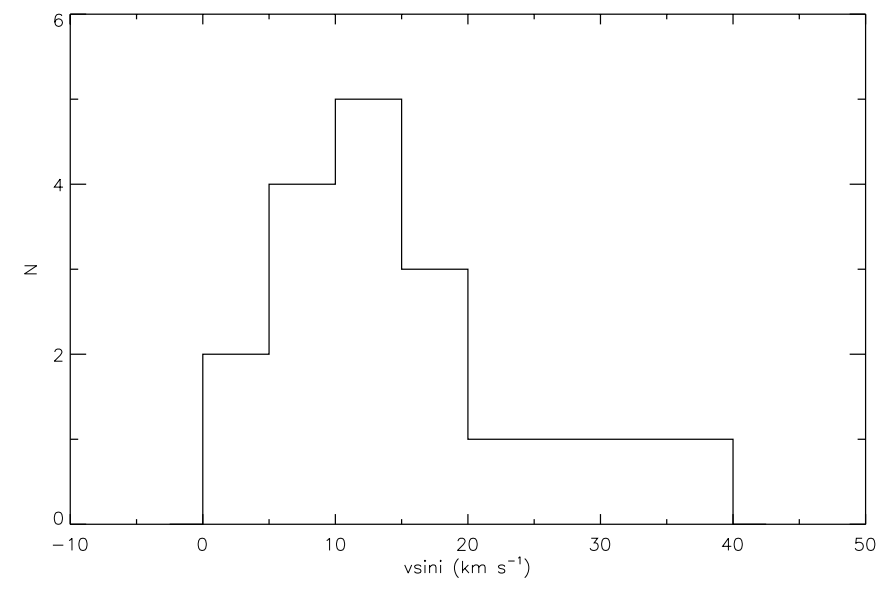

Fig. 3. $v \sin i_{\star}$ distribution of the 18 CoRoT-11-like stars from the compilation of Valenti \& Fischer (2005).

expected for a F6 V star (Gandolfi et al. 2010). The low activity level of CoRoT-11 is further confirmed by the lack of detectable spot-induced signatures in the composite stellar line profiles (cf. Fig. 1).

We searched for possible evidence of an equator-on view of CoRoT-11 by comparing its $v \sin i_{\star}$ with the projected rotational velocity of a sample of randomly oriented stars similar to CoRoT-11. For this purpose, we used the compilation of Valenti $\&$ Fischer (2005), which provides the projected rotational velocity for 1040 field stars. Figure 3 shows the $v \sin i_{\star}$ distribution of the sub-sample of 18 F-type stars, whose parameters resemble those of CoRoT-11. Although affected by a small-number statistics, the histogram seems to be asymmetric and peaks between $10 \mathrm{~km} \mathrm{~s}^{-1}$ and $15 \mathrm{~km} \mathrm{~s}^{-1}$. With a projected rotational velocity of $38.47 \pm 0.07 \mathrm{~km} \mathrm{~s}^{-1}$, CoRoT-11 lies in the very right tail of the distribution, suggesting that $i_{\star}$ is likely to be close to $90^{\circ}$.

A constraint on the true obliquity $\psi$ can be obtained from the variation in the duration of the transits of CoRoT-11. Since the star is rapidly rotating, its sizeable quadrupole moment induces a precession of the lines of the nodes of the orbital plane on a timescale as short as $\sim 7 \times 10^{4}$ years (Lanza et al. 2011). This produces a variation in the duration of the transits that can be used to constrain the obliquity. The effect is significant even for small obliquities $\psi<10^{\circ}$ and is worth searching for in the near future (Damiani \& Lanza 2011).

Our determination of $\lambda$ is consistent with the system currently being nearly aligned in the plane of the sky. This makes CoRoT-11 an interesting exception to the empirical rule stating that stars with $T_{\text {eff }} \geq 6250 \mathrm{~K}$ and $M_{\star} \geq 1.2 M_{\odot}$ have high obliquities (Winn et al. 2010). Lanza et al. (2011) pointed out that the tidal interaction timescale of the system is comparable to the estimated age of CoRoT-11 $(2 \pm 1 \mathrm{Gyr})$ if the average modified tidal quality factor of the star is $4 \times 10^{6} \lesssim\left\langle Q_{\star}^{\prime}\right\rangle \lesssim 2 \times 10^{7}$. One might thus wonder whether the orbit of the planet was initially highly tilted with respect to the stellar spin axis and the strong tidal torques then gradually aligned the system, damping down any initial misalignment.

To answer this question, we studied the tidal evolution of the obliquity, following the initial condition scenario described in Lanza et al. (2011). According to this scenario, on the zero-age main sequence (ZAMS) the planet would have had an initial orbital period that was nearly synchronous with the stellar spin. We assumed that $i_{\star}=i_{\mathrm{p}}=83.41^{\circ}$ (i.e., $\psi \approx \lambda$ ), which implies 


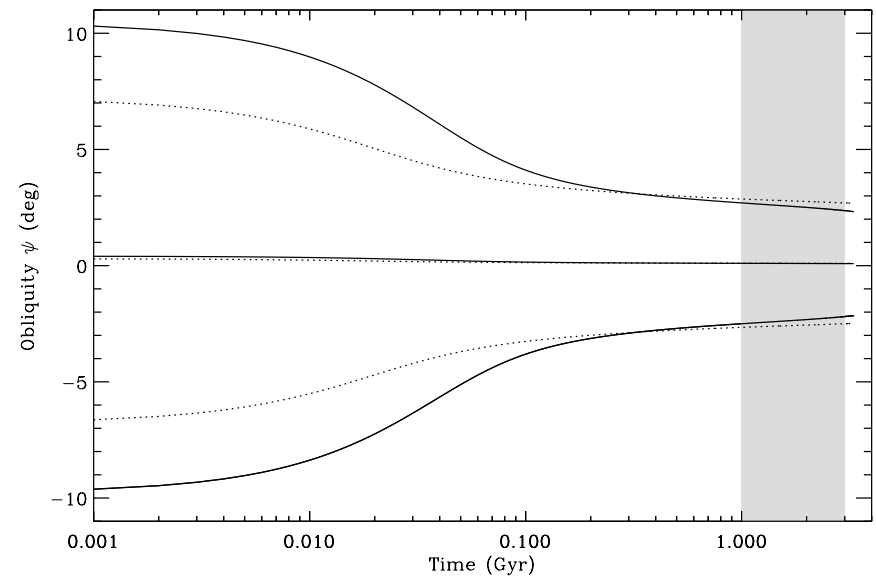

Fig. 4. Forward tidal evolution of the obliquity $\psi$ for $\left\langle Q_{\star}^{\prime}\right\rangle=4 \times 10^{6}$ (solid lines) and $\left\langle Q_{\star}^{\prime}\right\rangle=2 \times 10^{7}$ (dotted lines). The grey shaded area indicates the age of the star $(2 \pm 1 \mathrm{Gyr})$.

a current value of the stellar rotation period of $P_{\text {rot }}=1.74$ days. Figure 4 shows the forward tidal evolution of the true obliquity $\psi$ in the $\left\langle Q_{\star}^{\prime}\right\rangle$ domain found by Lanza et al. (2011) for the CoRoT-11 system, namely, $4 \times 10^{6} \lesssim\left\langle Q_{\star}^{\prime}\right\rangle \lesssim 2 \times 10^{7}$. With a system age of $2 \pm 1 \mathrm{Gyr}$, the current $\lambda=0.1^{\circ} \pm 2.6^{\circ}$ translates into an initial obliquity $|\psi| \lesssim 10^{\circ}$ on the ZAMS. We remind the reader that $\left\langle Q_{\star}^{\prime}\right\rangle \lesssim 4 \times 10^{6}$ would imply a non-synchronous initial state of the system, as well as an age younger than $1 \mathrm{Gyr}$ (Lanza et al. 2011). This is in contrast to the estimated age $(2 \pm 1 \mathrm{Gyr})$. On the other hand, if the star-planet tidal interaction were weak, i.e., $\left\langle Q_{\star}^{\prime}\right\rangle \gtrsim 2 \times 10^{7}$, the system would basically not have evolved during its lifetime and the current low obliquity would be close to that of its initial state on the ZAMS. The possibility that $\left\langle Q_{\star}^{\prime}\right\rangle$ might be as small as $10^{6}$ in the case of obliquity evolution, while of the order of $10^{7}-10^{9}$ in the case of planet migration was suggested by Lai (2012) and leads to results similar to those shown in Fig. 4, pointing again towards an initially low obliquity. Finally, an initially high eccentric orbit of CoRoT-11b would change the above results. However, this circumstance was discussed in detail in Sects. 3.3.1 and 4.2 of Lanza et al. (2011) and seems to be unlikely.

In conclusion, our results suggest a smooth, non-violent inward migration of CoRoT-11b during the system formation, involving angular momentum exchanges with the protoplanetary disc (e.g., Lin et al. 1996), which would not have perturbed the planet's primordial alignment.
Acknowledgements. We thank the editor and the anonymous referee for their careful reading, useful comments, and suggestions, which helped to improve and strengthen the manuscript. Part of the data presented herein were obtained at the W. M. Keck Observatory from telescope time allocated to the National Aeronautics and Space Administration through the agency's scientific partnership with the California Institute of Technology and the University of California. The Observatory was made possible by the generous financial support of the W. M. Keck Foundation. The authors wish to recognize and acknowledge the very significant cultural role and reverence that the summit of Mauna Kea has always had within the indigenous Hawaiian community. We are most fortunate to have the opportunity to conduct observations from this mountain.

\section{References}

Claret, A. 2004, A\&A, 428, 1001

Collier Cameron, A., Bouchy, F., Hébrard, G., et al. 2007, MNRAS, 375, 951

Collier Cameron, A., Bruce, V. A., Miller, G. R. M., et al. 2010a, MNRAS, 403, 151

Collier Cameron, A., Guenther, E., Smalley, B., et al. 2010b, MNRAS, 407, 507

Damiani, C., \& Lanza, A. F. 2011, A\&A, 535, A116

Donati, J. F., Semel, M., Carter, B. D., et al. 1997, MNRAS, 291, 658

Enoch, B., Collier Cameron, A., Parley, N. R., et al. 2010, A\&A, 516, A33

Fabrycky, D., \& Tremaine, S. 2007, ApJ, 669, 1298

Gandolfi, D., Hébrard, G., Alonso, R., et al. 2010, A\&A, 524, A55

Gaudi, B. S., \& Winn, J. N. 2007, ApJ, 655, 550

Giménez, A. 2006, ApJ, 650, 408

Kozai, Y. 1962, AJ, 67, 591

Kurucz, R. L. 1979, ApJS, 40, 1

Lai, D. 2012, MNRAS, 423, 486

Lanza, A. F., Damiani, C., \& Gandolfi, D. 2011, A\&A, 529, A50

Lin, D. N. C., Bodenheimer, P., \& Richardson, D. C. 1996, Nature, 380, 606

Mandel, K., \& Agol, E. 2002, ApJ, 580, 171

McLaughlin, D. B. 1924, ApJ, 60, 22

Miller, G. R. M., Collier Cameron, A., Simpson, E. K., et al. 2010, A\&A, 523, A52

Morton, T. D., \& Johnson, J. A. 2011, ApJ, 729, 138

Moutou, C., Diaz, R. F., Udry, S., et al. 2011, A\&A, 533, A113

Naoz, S., Farr, W. M., Lithwick, Y., et al. 2011, Nature, 473, 187

Ohta, Y., Taruya, A., \& Suto, Y. 2005, ApJ, 622, 1118

Pollacco, D., Skillen, I., Collier Cameron, A., et al. 2008, MNRAS, 385, 1576

Queloz, D., Eggenberger, A., Mayor, M., et al. 2000, A\&A, 359, L13

Rasio, F. A., \& Ford, E. B. 1996, Science, 274, 954

Rossiter, R. A. 1924, ApJ, 60, 15

Schlaufman, K. 2010, ApJ, 719, 602

Shporer, A., Bakos, G. A., Bouchy, F., et al. 2009, ApJ, 690, 1393

Southworth, J. 2011, MNRAS, 417, 2166

Torres, G., Andersen, J., \& Giménez, A. 2010, A\&ARv, 18, 67

Triaud, A. H. M. J. 2011, A\&A, 534, L6

Triaud, A. H. M. J., Collier Cameron, A., Queloz, D., et al. 2010, A\&A, 524, A25

Valenti, J. A., \& Fischer, D. 2005, ApJS, 159, 141

Vogt, S. S., Allen, S. L., \& Bigelow, B. C., et al. 1994, SPIE, 2198, 362

Winn, J. N. 2010, in Exoplanets, ed. S. Seager (University of Arizona Press), 55

Winn, J. N., Fabrycky, D., Albrecht, S., et al. 2010, ApJ, 718, L145

Wu, Y., Murray, N. W., \& Ramsahai, J. M. 2007, ApJ, 670, 820 